\title{
Integrated Risk Estimation in Fujiang River Basin in Sichuan by Using Information Diffusion Technology
}

\author{
Xiaojun Pan ${ }^{1,2,3,4}$, Chongfu Huang ${ }^{1,2,3, *}$, Lingyan Zhang ${ }^{1,2,3}$ \\ ${ }^{1}$ Key Laboratory of Environmental Change and Natural Disaster, Ministry of Education, Beijing Normal \\ University, Beijing 100875, China \\ ${ }^{2}$ State Key Laboratory of Earth Surface Processes and Resource Ecology, Beijing Normal University, Beijing \\ 100875, China \\ ${ }^{3}$ Faculty of Geographical Science, Academy of Disaster Reduction and Emergency Management, Beijing Normal \\ University, Beijing 100875, China \\ ${ }^{4}$ School of Economics, Southwest University for Nationalities, Chengdu610004, China

\section{基于信息扩散技术的四川涪江流域综合风险评估} \\ 潘小军 $^{1,2,3,4}$, 黄崇福 ${ }^{1,2,3, *}$, 张领雁 ${ }^{1,2,3}$ \\ '北京师范大学, 环境演变与自然灾害教育部重点实验室, 北京 100875, 中国 \\ ${ }^{2}$ 北京师范大学, 地表过程与资源生态国家重点实验室, 北京 100875 , 中国 \\ ${ }^{3}$ 北京师范大学, 地理科学学部, 减灾与应急管理研究院, 北京 100875 , 中国 \\ ${ }^{4}$ 西南民族大学, 经济学院, 成都 610004 , 中国
}

\begin{abstract}
The earthquake and flood damage data of the families in villages and towns along Fujiang River in Sichuan are collected by using Internet of intelligences. The information diffusion technology is used to estimate the integrated risk caused by earthquake and flood. The villages and towns have suffered a large loss of integrated risk caused by the two hazards, with an average annual loss of 5.758 million Yuan. Through the comprehensive suppression and blocking intervention combined with the village watershed management and post-disaster reconstruction, the
\end{abstract}

基金项目：本文为中央高校项目（2014SZYTD01）、国 家社科基金（16BJL115）、国家自然科学基金项目 (41671502) 等项目的阶段性研究成果。

作者简介：潘小军，西南民族大学经济学院、北京师范 大学减灾研究院博士研究生 1002343190@QQ.com, Tel:13261666399 黄崇福, 北京师范大学, 环境演变与自然灾 害教育部重点实验室教授、博士生导师。 张领雁, 北京师范大学, 环境演变与自然灾 害教育部重点实验室、硕士研究生。

*通讯作者: hchongfu@bnu.edu.cn frequency and the degree of disaster occurrence have gradually decreased in the past decades.

Keywords: Earthquake, Flood, Fujiang, Integrated risk, Information diffusion, Internet of intelligences

\section{摘要}

利用智联网收集四川涪江沿线乡镇以家庭为单元 的地震和洪水灾害数据, 并用信息扩散技术依此估 计地震和洪水综合风险。这些乡镇面临此两种灾害 较大的综合风险, 年均期望损失高达 575.8 万元。 流域治理与灾后重建结合的综合抑制阻断干预，已 使灾害发生频度与灾损程度近 10 年来逐渐降低。

关键词: 地震, 洪水, 涪江, 综合风险, 信息扩散, 智联网

\section{1. 问题提出}

如果没有相应的致灾环境, 风险因子也不可能 发育到损害风险载体的程度; 如果承载体的脆弱性 很低, 足以抵挡一定风险环境下致灾因子突变的损 害, 也未必会发生风险事件; 由于风险载体足够强 大，即使发生风险事件了，也未必有严重的风险损 失; 如果没有风险主体承担损失的后果, 则整个风 险要素顺序推进的结果只能说是自然运动现象 (荒 
漠中的自然活动是谈不上灾害或损失的)。显然, 灾害是一种或多种致灾因子在某一具体时空环境 中逐渐发育累积后偶然突发的客观致损事件, 更是 与人类相关的生命财产损害、生态环境变迁和经济 社会破坏的复杂时空异变过程。

现有灾害评估技术方法不能真实反映风险发 育的灾变过程不确定性与风险涌现突变的复杂性、 多变性和多样性, 以及风险转移管理活动的实际, 也难以跟上风险管理技术发展的要求。特别是, 在 全球气候变化加剧和地壳运动活跃下, 极端性灾害 频发和并发, 损害之大和救灾之难极为罕见, 对多 灾种应急处置的防灾减灾建设要求越来越高, 从多 灾种混合风险的综合治理的视角, 构建多灾种综合 风险管理对策模式, 始终是理论与技术实践的重要 突破方向。

\section{2. 文献综述}

20 世纪 80 年代联合国发起的自然灾害减灾行 动 (IDNDR) 到 21 世纪减轻灾害国际战略 (ISDR) 和减轻灾害风险 (DRR) 的变化, 推动了灾害风险 科学的研究与实践。多灾种混合风险的综合损害评 估的视角、理念和技术的文献比较多。全球或区域 性机构主导的大时空尺度的灾害风险综合评估技 术方法是主流联合国开发计划署 2004 年提出了世 界性的灾害风险指标计划 ${ }^{[1][2]}$, 美国哥伦比亚大学 和世界银行协作进行过区域性联合机构的多发展 指标计划 ${ }^{[3][4]}$ 及自然灾害风险管理指标系统美洲计 划 ${ }^{[5][6]}$ 。欧洲关注综合自然类和技术类致灾因子引 起风险的多重风险评估 ${ }^{[7]}, 2004$ 年, 美国联邦紧急 管理管理局和国家建筑科学院主导了的灾害评估 的 HAZUS 模型 ${ }^{[8]}$ 。

中国特色的各种时空尺度的灾害风险综合评 估技术方法不断涌现中国灾害分类与专业管理 ${ }^{[9]}$ (史培军、1991; 马宗晋等, 1998), 中国灾害综 合活动程度和综合危害程度图 ${ }^{[9]}$ (马宗晋等, 1998), 中国重大灾害风险多灾种区域安全预估 ${ }^{[10]}$ (高庆华 等, 1998），基于多灾种强度和城市化水平评估制 定中国县级城市自然灾害区划图 ${ }^{[11][12]}$ (王静爱等), 市县村三级尺度的自然灾害综合风险评估 ${ }^{[13]}$ (葛全 胜等）。

用综合法对多灾种风险评估越来越多早期简 单的加权综合法从等权重 ${ }^{[14]}$ (慕尼黑再保险公司, 2003)、非等权重 ${ }^{[15]}$ (欧洲空间观测网络 (ESPON), 2004)、到层次分析法 (AHP) (Yoshimatsu ${ }^{[16]}$, 2006; 张继权 ${ }^{[17]}, 2007$; 费振宇等 $\left.{ }^{[187}, 2013\right) 、$ 概率统计

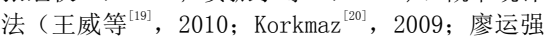
等 ${ }^{[21]}, 2014$ )、灰色系统法 ( 吴晓和吴宜进 ${ }^{[22]}, 2014$; 裴启涛等 ${ }^{[23]}, 2013$; 王伟等 ${ }^{[24]}$, (2014)、人工神 经网络法 (刘少华等 ${ }^{[25]}, 2013$ )、地理信息系统与 遥感技术 (GIS\&RS)、信息扩散法 (黄崇福等 ${ }^{[26]}, 2014$; 吴肜等 ${ }^{[27]}$, 2014; 郭君等 ${ }^{[28]}$, 2015) 等, 既有把多 灾种风险理解为区域内多种风险的简单叠加 ${ }^{[29]}$, 考 虑了致灾因子之间关系 ${ }^{[30]}$, 又有自然灾害和事故灾 难耦合的风险评估方法 ${ }^{[31]}$, 也有不考虑致灾因子间 因果触发关系只单独归为灾害链问题 ${ }^{[32]}$ 。显然, 多
灾种综合风险分析技术方法的不断创新和丰富, 让 认识了解多灾种综合风险及其治理方案设计更加 科学和容易。

但是, 在灾害风险评价中小尺度上存在数据不 完备性产生较大误差, 影响风险评估的准确性, 如 何克服样本容量不足与历史信息缺失的问题, 得到 较为可靠的分析结论, 是多灾种综合风险评估的重 要方向, 小流域乡村农户家庭微观调查数据的多灾 种综合损害评估则是当前研究的难点。本文基于智 联网平台采集样本观测点遭受多灾种综合侵害的 微观数据, 运用信息扩散技术对大时空尺度下观测 点农户家庭生产与生活遭受侵害的程度进行评估, 从多灾种混合风险的综合治理的视角, 构建多灾种 综合风险管理对策模式。

\section{3. 研究区域概况及数据来源 \\ 3. 1 研究区域}

本文选取三台县涪江流域某乡镇作为研究样 本点, 研究涪江流域综合风险。

涪江发源于四川省松潘县境内戉山主峰雪宝 顶,东北坡三岔子,涪江过绵阳市区后,进入绵阳市的 三台县境,转向东南流, 经芦溪镇、花园镇、刘营镇、 灵兴镇、新德镇, 县城潼川镇, 在潼川镇东塔山下, 接纳右岸支流凯江, 经三台县百顷入射洪县境.

涪江沿岸人口稠密, 城镇密集, 沿干流仅乡以 上集镇即达 80 余个。涪江洪水频繁, 易造成干流的 流域性洪灾, 据史料记载:公元 277 年以来的 1000 余年中,涪江曾发生过上百次洪水, 仅 1744 至 1949 年间, 就出现过地跨上中下游的流域性大洪水 15 次, 平均不到 14 年即发生一次。近 500 年来, 涪 江沿岸的 15 座城镇中,干流 7 座, 支流 6 座城市曾 被淹没过; 近 200 年间, 沿江县以上城市遭受洪灾 50 城市次, 干流上的绵阳、三台、射洪、遂宁、潼 南五城即遭洪灾达 32 次。

涪江所在的四川也是我国大陆地震多发区之 一。据史料记载:从公元前 26 年至 2008 年 5 月 26 日, 计发生 5 级以上地震 272 次, 其中 5 5.9 级地震 195 次, 6 6.9 级地震 57 次, 7 7.9 级地震 19 次, 8.0 级地震 1 次。

\section{2 数据来源}

通过走访灾害相关管理部门, 与乡镇农技员座 谈; 借助于智联网平台, 用随机抽样的方式, 通过 入户调查获取第一手的数据, 结合历年的《三台县 志》, 《县统计年鉴》; 《县统计公告》; 《县民族统计 报表》及相关乡镇的乡志; 以及县麦冬办、灾后重 建办公室、镇乡政府的农办和民政办提供的资料。

\section{4. 信息扩散技术模型构建}

\section{1 基于信息扩散地震洪水综合风险分析}

4.1.1. 地震、洪水致灾因子强度

\section{（1）洪水致灾因子}

用 1958-2015 气象降雨数据,计算年均降雨量, 据公式 (1) - (3) 计算出的 Z 指数作为洪涝致灾 因子的强度（见表 1)。 


$$
\begin{gathered}
z_{j}=\frac{6}{C_{s}} \times\left(\frac{C_{s} \emptyset_{j}}{2}+1\right)^{\frac{1}{3}}-\frac{6}{C_{s}}+\frac{C_{s}}{6} \\
C_{s}=\frac{\sum_{j=1}^{n}\left(p_{j}-\bar{p}\right)^{3}}{n \sigma^{3}} \\
\emptyset_{j}=\frac{p_{j}-\bar{p}}{\sigma}
\end{gathered}
$$

式中,

$Z_{j}-$--第 $j$ 年的 $Z$ 指数

$C_{s}$---偏态系数;

$\Phi_{j}---$ 降水量标准化变量;

$P_{j}--$-第 $j$ 年降水量;

$n$---系列长度;

$P--$ 降水系列平均值;

$\sigma---$ 系列标准差。

表 1: 洪涝灾害 Z 指数及旱涝等级

\begin{tabular}{ccc|ccc}
\hline 年份 & $Z$ 指数 & 程度 & 年份 & $Z$ 指数 & 程度 \\
\hline 1963 & 1.49 & 大涝 & 1989 & 0.91 & 偏涝 \\
1964 & 1.22 & 大涝 & 1998 & 1.54 & 大涝 \\
1973 & 1.60 & 大涝 & 2000 & 1.43 & 大涝 \\
1980 & 0.92 & 偏涝 & 2011 & 1.25 & 大涝 \\
1981 & 2.55 & 重涝 & 2013 & 1.17 & 大涝 \\
1983 & 1.64 & 大涝 & & & \\
\hline
\end{tabular}

\section{（2）地震致灾因子}

地震灾害损失评估包括人员伤亡、地震造成的 经济损失以及建筑物破坏状况评估。经济损失是指 地震及其场地灾害、次生灾害造成的建筑物和其他 工程结构、设施、设备、财物等破坏而引起的经济 损失。对于地震灾害的损失我们借助于历年的《县 的统计年鉴》、《统计公报》并结合随机抽样评估地 震损失, 评估出地震风险的大小。

\subsection{2. 地震和洪水的综合损失评估}

根据调研数据计算出平均损失 $\times$ 户数作为历史 最严重洪涝损失, 根据指数, 当时的社会经济发展 水平估算出洪涝灾害导致的财产损失，根据同样的 方法估计出地震灾害损失。进一步估计出地震和洪 水的综合损失 (见表 2)。

\section{2 洪水、地震损失与综合风险的评估}

虽然我们使用智联网平台, 利用随机抽样方式 入户调查来获取数据, 但仅这些信息对于全面认识 相关流域的地震、洪水综合风险是不够的, 所以我 们进一步使用信息扩散的方法, 将调研获得的数据 在空间进行信息扩散, 较好的估计地震、洪水的各 自的经济损失和风险, 以及地震和洪水造成的综合 的经济损失和风险。

信息扩散理论: 针对自然灾害风险分析领域经 常遇到不完备信息的这一情况, 黄崇福等经过长期 的试验、验证以及多领域的应用研究系统地提出了 一套基于模糊集理论,处理不完备信息的理论技术, 即信息扩散技术。信息扩散技术主要包括信息矩阵、 信息分配和信息扩散三个部分。

\begin{tabular}{ccccc}
\multicolumn{6}{c}{ 表 $2:$ 洪涝地震灾害的强度和损失 (万元) } \\
\hline 洪涝 & 洪涝财 & 地震 & 震害 & 总损 \\
指数 & 产损失 & 震级 & 损失 & 失 \\
\hline 1.49 & 8.7 & 0 & 0 & 8.7 \\
1.22 & 2.3 & 0 & 0 & 2.3 \\
1.6 & 13.4 & 0 & 0 & 13.4 \\
0.92 & 1.2 & 0 & 0 & 1.2 \\
2.55 & 62.3 & 0 & 0 & 62.3 \\
1.64 & 50.6 & 0 & 0 & 50.6 \\
0.91 & 2.1 & 0 & 0 & 2.1 \\
1.54 & 1575 & 0 & 0 & 1575 \\
1.43 & 20.8 & 0 & 0 & 20.8 \\
0 & 0 & 8 & 2270 & 2270 \\
0 & 0 & 5.2 & 500 & 500 \\
0 & 0 & 5.2 & 123.1 & 123 \\
0 & 0 & 5.7 & 234.1 & 234 \\
0 & 0 & 5.2 & 100.4 & 100 \\
0 & 0 & 6.1 & 1232 & 1232 \\
0 & 0 & 6 & 1023 & 1023 \\
0 & 0 & 5.6 & 305.2 & 305 \\
1.25 & 9.3 & 0 & 0 & 9.3 \\
1.17 & 10.5 & 4.5 & 23.4 & 33.9 \\
0 & 0 & 4.9 & 30.2 & 30.2 \\
\hline
\end{tabular}

信息扩散是一种对样本进行集值化的模糊数 学处理方法, 它可以将单值样本变成集值样本最 简单的模型是正态扩散模型。

\subsection{1. 一维信息扩散 \\ 采样样本集为:}

$$
Y=\left\{y_{1}, y_{2}, \cdots, y_{m}\right\}
$$

设灾害指标论域为:

$$
U=\left\{u_{1}, u_{2}, \cdots, u_{n}\right\}
$$

式中, $u_{1}, u_{2}, \cdots, u_{n}$ 为控制点。

一个单值观测样本点 $y_{j}$ 可以将其所携带的信 息扩散给 $U$ 中的所有点。

$$
f\left(u_{i}\right)=\frac{1}{h \sqrt{2 \pi}} \exp \left[-\frac{\left(y_{j}-u_{i}\right)^{2}}{2 h^{2}}\right], i=1,2, \cdots, n
$$

$h$ 为扩散系数。设 $a, b$ 为 $Y$ 中最大, 最小值, 则:

$$
\begin{aligned}
& h= \begin{cases}1.6987(b-a) /(m-1) & 1<m \leqslant 5 \\
1.4456(b-a) /(m-1) & 6 \leqslant m \leqslant 7 \\
1.4230(b-a) /(m-1) & 8 \leqslant m \leqslant 9 \\
1.4208(b-a) /(m-1) & 10 \leqslant m\end{cases} \\
& \text { 令 } C_{j}=\sum_{i=1}^{n} f_{j}\left(u_{i}\right)
\end{aligned}
$$

则相应的模糊子集的隶属函数为:

整理后为:

$$
\mu_{y_{j}}\left(u_{i}\right)=\frac{f_{j}\left(u_{i}\right)}{C_{j}}
$$

$$
q\left(u_{i}\right)=\sum_{j=1}^{m} \mu_{y_{j}}\left(u_{i}\right)
$$

式中, $m$ 为观测样本点个数。再令:

$$
Q=\sum_{i=1}^{n} q\left(u_{i}\right)
$$

理论上讲 $Q=m$ 。所以 $p\left(u_{-} i\right)=q\left(u_{-} i\right) / Q$

就是样本落在 $u_{i}$ 处的频率值, 可作为概率的估 计值。显然超过 $u_{i}$ 的概率值应为: 


$$
P\left(u_{i}\right)=\sum_{k=i}^{n} p\left(u_{k}\right)
$$

\subsection{2.二维信息扩散}

设 $X$ 是一给定样本, 含有 $n$ 个样本点, 每个样 本点有两个分量, 分别是输入值 $x$ 和输出值 $y$ 。该 样本记为: $X=\left\{\left(x_{1}, y_{1}\right),\left(x_{2}, y_{2}\right), \ldots,\left(x_{n}, y_{n}\right)\right\}$ 。假设某个客 观事物的全貌 $R$ 是通过 $X$ 来认识, 称 $X$ 提供了认 识 $R$ 的信息。当 $X$ 提供的信息不足以认识 $R$ 时, 我们称 $X$ 是认识 $R$ 的不完备信息, 简称信息不完备。 当 $X$ 中的样本点过少时, 就称为小样本问题。

当将两灾种 $S$ 和 $Z$ 的综合风险界定为其在 $T$ 年 内造成灾害 $D$ 的期望值时, 综合风险评估的工作就 是:

估计 $S$ 和 $Z$ 的联合概率分布 $p(s, z)$, 估计脆弱 性函数 $d=f(s, z)$, 则有

$$
\text { Risk }=\int_{s_{0}}^{s_{\theta}} \int_{z_{o}}^{z_{\theta}} f(s, z) p(s, z) d s d z
$$

$s_{0}$---能造成灾害的灾种 $S$ 的最小值

$s_{\theta}-$-研究区域灾种 $S$ 可能出现的最大值

$z_{0}$---能造成灾害的灾种 $Z$ 的最小值

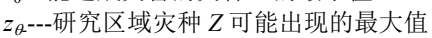

特别地，假定每年关于 $S$ 和 $Z$ 各有一条记录, $T$ 年内得到的两个变量的观测样本为:

$$
W_{1}=\left\{\left(s_{1}, z_{1}\right),\left(s_{2}, z_{2}\right), \ldots,\left(s_{T}, \mathrm{z}_{T}\right)\right\}
$$

用 $S$ 和 $Z$ 分别表达两灾种的离散论域:

$$
\left\{\begin{array}{c}
S=\left\{u_{j} \mid j=1,2, \cdots, J\right\} \\
Z=\left\{v_{k} \mid k=1,2, \cdots, K\right\}
\end{array}\right.
$$

用 2 维正态扩散将 $W_{1}$ 在 $S \times Z$ 上进行扩散处理

$$
\mu\left(\left(\mathrm{s}_{i}, \mathrm{z}_{i}\right),\left(\mathrm{u}_{j}, \mathrm{v}_{k}\right)\right)=\exp \left\lfloor-\frac{\left(\mathrm{s}_{i}-\mathrm{u}_{j}\right)^{2}}{2 h_{s}^{2}}\right\rfloor \exp \left[-\frac{\left(z_{i}-v_{k}\right)^{2}}{2 h_{z}^{2}}\right]
$$

$\forall g \in\{s, z\}$, 扩散系数 $h_{g}$ 的计算公式为:

$$
\begin{aligned}
& \left(\begin{array}{l}
0.8146\left(b_{g}-a_{g}\right), \quad T=5 ; \\
0.5690\left(b_{g}-a_{g}, \quad T=6\right.
\end{array}\right. \\
& h_{g}= \begin{cases}0.5690\left(b_{g}-a_{g}\right), & T=6 ; \\
0.4560\left(b_{g}-a_{g}\right), & T=7 ; \\
0.3860\left(b_{g}-a_{g}\right), & T=8 ; \\
0.3362\left(b_{g}-a_{g}\right), & T=9 ; \\
0.2986\left(b_{g}-a_{g}\right), & T=10 ; \\
2.6851\left(b_{g}-a_{g}\right) /(T-1), & T \geq 11 .\end{cases} \\
& b_{g}=\max _{1<i<T}\left\{g_{i}\right\}, \quad a_{g}=\min _{1 \leq i<T}\left\{g_{i}\right\} .
\end{aligned}
$$

从而可估计得联合概率分布:

$$
P\left(u_{j}, v_{k}\right)=\frac{\sum_{i=1}^{T} \mu\left(\left(s_{i}, z_{i}\right),\left(u_{j}, v_{k}\right)\right)}{\sum_{j=1}^{J} \sum_{k=1}^{K} \sum_{i=1}^{T} \mu\left(\left(s_{i}, z_{i}\right),\left(u_{j}, v_{k}\right)\right)}
$$

令:

$$
Q_{j k}=\sum_{i=1}^{T} \mu\left(\left(\mathrm{s}_{i}, \mathrm{z}_{i}\right),\left(\mathrm{u}_{j}, \mathrm{v}_{k}\right)\right)
$$

得信息矩阵:

$$
Q=\begin{gathered}
u_{1} \\
u_{2} \\
\cdots \\
u_{J}
\end{gathered}\left(\begin{array}{cccc}
v_{1} & v_{2} & \cdots & v_{K} \\
Q_{11} & Q_{12} & \cdots & Q_{1 K} \\
Q_{21} & Q_{22} & \cdots & Q_{2 K} \\
\cdots & \cdots & \cdots & \cdots \\
Q_{J 1} & Q_{J 2} & \cdots & Q_{J K}
\end{array}\right)
$$

\subsection{3.三维信息扩散}

用包含灾害信息的整个观测样本

$$
W=\left\{\left(s_{1}, z_{1}, d_{1}\right),\left(s_{2}, z_{2}, d_{2}\right), \cdots,\left(s_{T}, z_{T}, d_{T}\right)\right\}
$$

估计脆弱性函数。 $S$ 和 $Z$ 为两灾种的论域, $D$ 为财 产损失的论域

$$
\left\{\begin{array}{l}
S=\left\{u_{j} \mid j=1,2, \cdots, J\right\} \\
Z=\left\{v_{k} \mid k=1,2, \cdots, K\right\} \\
D=\left\{o_{l} \mid l=1,2, \cdots, L\right\}
\end{array}\right.
$$

用 3 维正态扩散将 $W$ 在 $S \times Z \times D$ 上进行扩散处理

$$
\begin{aligned}
& \mu\left(\left(s_{i}, z_{i}, d_{i}\right),\left(u_{j}, v_{k}, o_{l}\right)\right) \\
& =\exp \left[-\frac{\left(s_{i}-u_{j}\right)^{2}}{2 h_{s}^{2}}\right] \exp \left[-\frac{\left(z_{i}-v_{k}\right)^{2}}{2 h_{z}^{2}}\right] \exp \left[-\frac{\left(d_{i}-o_{l}\right)^{2}}{2 h_{d}^{2}}\right]
\end{aligned}
$$

令:

$$
Q_{j k l}=\sum_{i=1}^{T} \mu\left(\left(s_{i}, z_{i}, d_{i}\right),\left(u_{j}, v_{k}, o_{l}\right)\right)
$$

得信息矩阵:

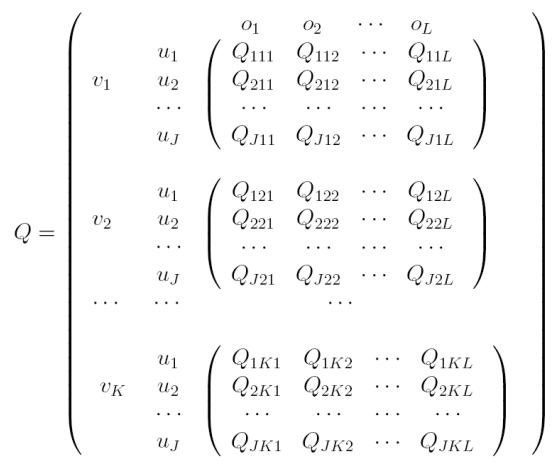

$\forall l \in\{1,2, . ., L\}$, 令:

$$
H_{l}=\max _{\substack{1 \leq j \leq J \\ 1 \leq k \leq K}}\left\{Q_{j k l}\right\}
$$




$$
\begin{gathered}
r_{j k l}=\frac{Q_{j k l}}{H_{l}}, \\
j=1,2, \cdots, J ; k=1,2, \cdots, K
\end{gathered}
$$

输入为 $(S, Z)$, 输出为 $d$ 间的模糊关系矩阵:

$$
R=\left\{r_{j k l}\right\}_{J \times K \times L}
$$

对于具有隶属函数 $\mu_{\mathrm{A}}\left(\mathrm{u}_{j}, \mathrm{v}_{k}\right)$ 的模糊输入 $A$, 近似推进公式, 可得隶属函数为 $\mu_{B}\left(o_{1}\right)$, $D$ 的模糊输出 $B$ :

$\iota_{\mathrm{B}}\left(\mathrm{o}_{1}\right)=\max _{1 \leq \mathrm{j} \leq \mathrm{J}, 1 \leq \mathrm{k} \leq \mathrm{K}} \min \left\{\mu_{\mathrm{A}}\left(\mathrm{u}_{\mathrm{j}}, \mathrm{v}_{\mathrm{k}}\right), \mathrm{r}_{\mathrm{jk} 1}\right\}$

最后, 用重心法得到一个分明值:

$$
\mathrm{d}\left(\mathrm{u}_{\mathrm{j}}, \mathrm{v}_{\mathrm{k}}\right)=\left(\sum_{\mathrm{l}=1}^{\mathrm{L}} \mu_{B}\left(\mathrm{O}_{L}\right) / \mathrm{O}_{L}\right) /\left(\sum_{\mathrm{l}=1}^{\mathrm{L}} \mu_{B}\left(\mathrm{O}_{L}\right)\right)
$$

它是用观测样本 $W$, 由三维正态信息扩散构造 个离散的脆弱性曲面。

\section{. 用三维信息扩散理论对样本点地震和洪水 风险评估}

利用智联网收集到的四川涪江沿线地震和洪 害数据共 25 个:

$\left.\left(s_{1}, z_{1}, d_{1}\right),\left(s_{2}, z_{2}, d_{2}\right), \ldots,\left(s_{25}, z_{25}, d_{25}\right)\right\}$ 49,0,8.7),(1.22,0,2.3),(1.6,0,13.4),(0.92,0,1.2),
$(2.55,0,62.3),(1.64,0,50.6),(0.91,0,2.1),(1.54,0,1575.4$ $8),(1.43,0,20.8),(0,8,2270.38),(0,5.2,500.3),(0,5.2,500$ $.3),(0,5.2,123.1),(0,5.7,234.1),(0,5.2,100.4),(0,6.1,123$ 2),(0,5.6,1.305.2),(1.25,0,9.3),(1.17,4.5,33.9),(0,4.9,3 $0.2)\}$

用 $S$ 和 $Z$ 分别表达洪水和地震的论域, 用 $D$ 表达财产损失的论域（万元）:

$$
\begin{aligned}
S= & \left\{u_{1}, u_{2}, \ldots, u_{11}\right\} \\
= & \{0,0.8,1,1.2,1.4,1.6,1.8,2,2.2,2.4,2.6\} \\
\mathrm{Z}= & \left\{v_{1}, v_{2}, \ldots, v_{8}\right\}=\{0,5,5.5,6,6.5,7,7.5,8\} \\
D= & \left\{o_{1}, o_{2}, \ldots o_{10}\right\}=\{0,100,200,300,400,500,1000, \\
& 1500,2000,2500\}
\end{aligned}
$$

计算得洪水地震离散联合概率分布 $P\left(u_{j}, v_{k}\right)$ (表 3$)$ 和洪水、地震下的离散脆弱性曲面 $d\left(u_{j}, v_{k}\right)$ (表 4$)$ 。由 它们可以计算出综合风险值为 575.8 万元。

\section{5. 研究结论和对策建议}

本文应用信息扩散方法计算出的涪江流域三 台县某乡镇观测点村庄遭受洪水和地震综合损失 较大, 平均每年达到 575.8 万元, 虽然有一些治理, 但依旧存在很多隐患, 能否应对大时间尺度比如 100 年一遇的洪水灾害就需要进一步的分析。研究 还发现, 观测点村庄多灾种综合灾害风险发育与传 递机理呈“一风险环境 (成灾环境) 一风险因素 (致

表3: 洪水地震离散联合概率分布

\begin{tabular}{l|llllllll}
\hline \multicolumn{1}{l}{} & $v_{1}=0$ & $v_{2}=5$ & $v_{3}=5.5$ & $v_{4}=6$ & $\mathrm{v}_{5}=6.5$ & $\mathrm{v}_{6}=7$ & $\mathrm{v}_{7}=7.5$ & $\mathrm{v}_{8}=8$ \\
\cline { 2 - 9 }$u_{1}=0.0$ & 0.003 & 0.067 & 0.073 & 0.067 & 0.052 & 0.036 & 0.023 & 0.016 \\
$u_{2}=0.8$ & 0.043 & 0.019 & 0.018 & 0.015 & 0.011 & 0.007 & 0.005 & 0.003 \\
$u_{3}=1.0$ & 0.059 & 0.013 & 0.011 & 0.008 & 0.005 & 0.003 & 0.002 & 0.001 \\
$u_{4}=1.2$ & 0.071 & 0.01 & 0.008 & 0.005 & 0.003 & 0.001 & 0.001 & 0 \\
$u_{5}=1.4$ & 0.075 & 0.008 & 0.006 & 0.003 & 0.002 & 0.001 & 0 & 0 \\
$u_{6}=1.6$ & 0.068 & 0.006 & 0.004 & 0.002 & 0.001 & 0 & 0 & 0 \\
$u_{7}=1.8$ & 0.054 & 0.003 & 0.002 & 0.001 & 0.001 & 0 & 0 & 0 \\
$u_{8}=2.0$ & 0.038 & 0.001 & 0.001 & 0.001 & 0 & 0 & 0 & 0 \\
$u_{9}=2.2$ & 0.025 & 0.001 & 0 & 0 & 0 & 0 & 0 & 0 \\
$u_{10}=2.4$ & 0.017 & 0 & 0 & 0 & 0 & 0 & 0 & 0 \\
$u_{11}=2.6$ & 0.013 & 0 & 0 & 0 & 0 & 0 & 0 & 0 \\
\hline
\end{tabular}

\begin{tabular}{l|crrrrrrr}
\hline \multicolumn{1}{c}{ 表 4: 洪水、地震下的离散脆弱性曲面 } \\
\hline & \multicolumn{1}{c}{$\mathrm{v}_{1}=0$} & \multicolumn{1}{c}{$\mathrm{v}_{2}=5$} & \multicolumn{1}{c}{$\mathrm{v}_{3}=5.5$} & $\mathrm{v}_{4}=6$ & $\mathrm{v}_{5}=6.5$ & $\mathrm{v}_{6}=7$ & $\mathrm{v}_{7}=7.5$ & $\mathrm{v}_{8}=8$ \\
\cline { 2 - 10 } $\mathrm{u}_{1}=0.0$ & 0 & 482.19 & 546.14 & 631.38 & 756.84 & 956.32 & 1247.7 & 1560.7 \\
$\mathrm{u}_{2}=0.8$ & 377.95 & 409.83 & 481.88 & 577.48 & 713.95 & 924.73 & 1228.3 & 1552.2 \\
$\mathrm{u}_{3}=1.0$ & 436.61 & 336.63 & 399.81 & 493.61 & 635.83 & 859.99 & 1185.3 & 1532.5 \\
$\mathrm{u}_{4}=1.2$ & 495.74 & 274.11 & 310.42 & 375.26 & 493.21 & 710.18 & 1065.1 & 1470.8 \\
$\mathrm{u}_{5}=1.4$ & 548.05 & 243.52 & 257.83 & 286.93 & 350.09 & 496.9 & 817.64 & 1301 \\
$\mathrm{u}_{6}=1.6$ & 587.69 & 232.56 & 237.23 & 247.33 & 271.28 & 336.27 & 524.41 & 964.52 \\
$\mathrm{u}_{7}=1.8$ & 608.67 & 229.1 & 230.49 & 233.65 & 241.41 & 263.85 & 339.09 & 587.44 \\
$\mathrm{u}_{8}=2.0$ & 600.36 & 228.08 & 228.42 & 229.37 & 231.75 & 238.77 & 263.66 & 360.32 \\
$\mathrm{u}_{9}=2.2$ & 546.4 & 227.82 & 227.8 & 228.07 & 228.78 & 230.92 & 238.6 & 270.22 \\
$\mathrm{u}_{10}=2.4$ & 446.86 & 227.81 & 227.62 & 227.68 & 227.89 & 228.53 & 230.85 & 240.58 \\
$\mathrm{u}_{11}=2.6$ & 345.5 & 227.91 & 227.58 & 227.57 & 227.62 & 227.82 & 228.51 & 231.45 \\
\hline
\end{tabular}


灾因子) 一风险载体 (承灾载体) 一风险事件 (致 灾事件) 一风险损失 (灾害损失) 一一险主体 (承 灾主体) 一”的规律。通过观测点村庄流域治理与 灾后重建结合的综合抑制阻断干预, 灾害发生频度 与灾损程度近 10 年来逐渐降低, 多灾种综合风险 管理模式效果显著, 对提高区域性综合灾害的分析 评估与预测能力, 强化事前防灾减灾方案设计效果, 减轻生命财产、生态环境和经济危害等有参考价值。

\section{参考文献}

[1] Pelhng M, Maskrey A, Ruiz P, et al. United Nations Development Program. A global report reducing disaster risk: A challenge for development. 1-146, 2004

[2] Pelling M, Visions of Risk: A Review of International Indicators of Disaster Risk and its Management. ISDR /UNDP: Kingps College, University of London, 1- 56,2004.

[3] Dilley M, Chen R S, Deichmann U, et al. Natural Disaster Hotspots: A Global Risk Analysis Washington DC: Hazard Management Unit, World Bank, 1- 132,2005.

[4] 薛华,陈报章,黄崇福,严建武. 多灾种综合风险评估软 层次模型. 地理科学进展, 2012, 31(3): 353-360.

[5] Cardona O. D, Hurtado J. E, Chardon A. C, et al. Indicators of disaster risk and risk management Main technical report. Program for Latin America and the Caribbean IADB - UNC / IDEA, World Bank, $1-216,2005$.

[6] Cardona O D, Hurtado J E, Chardon A C, et al Indicators of disaster risk and risk management Summary report for WCDR. Program for Latin America and the Caribbean IADB - UNC /IDEA, 1-47,2005.

[7] Grieving Ault-risk assessment of Europe's regions in: Beckmann Jed. Measuring Vulnerability to Hazards of National origin. Tokyo, UNU Press.210-226.

[8] FEMA. Using HAZUS-MH for Risk Assessment, http/www.fe nrn.gov/plan/prevent/hazus/dl fema4 33.shtm, 2004/2014-1-2

[9] 科技部国家计委国家经贸委灾害综合研究组, 广州地 理研究所.中国重大自然灾害与社会图集.广州：广 东科技出版社, 2004,1-120.

[10] 高庆华.中国自然灾害风险与区域安全性分析．北 京:气象出版社, 2005,1-130.

[11] 王静爱, 史培军, 王瑛, 等. 中国城市自然灾害区 划编制.自然灾害学报，2005，14(6):42-46。

[12] 王静爱, 史培军, 王平, 等. 中国自然灾害时空格 局. 北京: 科学出版社，2006,1-35.

[13] 葛全胜, 邹铭, 郑景云. 中国自然灾害风险综合评 估初步研究. 北京: 科学出版社, 2008,1-41.

[14] Munich Re . Topics-Annual Review: Natural Catastrophes 2002 . Munich, Germany. 2002, 21-33.
[15] P. Schmidt-Thom. The Spatial Effects and Management of Natural and Technological Hazards in Europe . Luxembourg: ESPON, 2006,11-16.

[16] Yoshimatsu H, Abe S. A review of landslide hazards in Japan and assessment of their susceptibility using an Analytical hierarchic process (AHP) method, landslide, 2006,3(2): 149-158.

[17] 张继权, 刘兴朋, 佟志军. 草原火灾风险评价与分区. 地理研究,2007,26(4):754-762,

[18] 费振宇, 周玉良等. 区域抗旱能力评价指标体系和 评价模型的构建..灾害学, 2013, 28(4): 197-204.

[19] 王威, 田杰等. 基于贝叶斯随机评价方法的小城镇灾 害易损性分析.防灾减灾工程学报,2010,36(5): 524-527.

[20] Korkmaz K A. Earthquake disaster risk assessment and evaluation for Turkey. Environmental Geology,2009, 57(2): 307-418.

[21] 廖远强,王斌会等.基于空间统计模型的热带气旋路 径模拟及其风险评估. 数理统计与管理, 2014,33(001): 31-41.

[22] 吴晓,吴宜进.基于灰色关联模型的山地城市生态安 全动态评价. 长江流域资源与环境, 2014, 23(3):385-390

[23] 裴启涛, 李海波等.基于改进的灰评估模型在岩爆中 的预测研究. 岩石力学与工程学报,2013,32(10): 2088-2093.

[24] 王伟, 雷晓辉等.石羊河流域中长期径流预报模型应 用.人民黄河，2014，36(1): 42-44.

[25] 刘少华,翁白莎等.农业干旱风险分析基本模式及应 用.水电能源科学 2013 (4): 5-8.

[26] 苏妩, 黄崇福等. 基于智联网的海洋环境风险评估 互联网平台初探.第二届中国沿海地区灾害风险分 析与管理学术研讨会,2014.11:14-20

[27] 吴䑣, 黄崇福等. 基于综合风险评估模式与信息扩 散原理的台风灾害风险评估方法探讨及应用.中国 灾害防御协会风险分析专业委员会第六届年会论 文集,2014.8: 698-703

[28] 郭君，黄崇福等. 与月份及预警有关的广东省台风 动态风险系统. 工程理论与实践, 2015（6): 1604-1616

[29] Dilley M. Natural disaster hotspots: a global risk analysis. World Bank Publications, 2005,1-33.

[30] Li Y, Lindt J W, Loss-based formulation for multiple hazards with application to residential buildings. Engineering Structures, 2012, 38( 1): 123-133.

[31] 盖程程, 翁文国, 袁宏永.基于 GIS 的多灾种耦合综 合风险评.清华大学学报(自科), 2011, 51(5)：627631 .

[32] Shi P, Shuai J, Chen W, et al. Study on large-scale disaster risk assessment and risk transfer models. International Journal of Disaster Risk Science, 2010, 1(2): 1-8 